\title{
Live Skin Allograft in the Management of Severe Burns
}

\author{
Shaban Saidi
}

\author{
Nyeri Provincial General Hospital
}

Correspondence to: Dr. Shaban Saidi, P.O Box 27 Nyeri. Email: sogshaban@yahoo.com

\begin{abstract}
Introduction: Deep burn injuries lead to dermal damage that impairs the ability of the skin to heal and regenerate on its own. Skin autografting is considered the current gold standard of care, but lack of patient's own donor skin may require the temporary use of skin substitutes to promote wound healing, reduce pain, and prevent infection and abnormal scarring. These alternatives include donor skin allograft, xenograft, cultured epithelial cells and biosynthetic skin substitutes. Skin allograft is the use of skin from a genetically non-identical member of the same species as the recipient. Human deceased or live donor skin allografts represent a suitable and much used temporizing option for skin cover following severe burn injury until autografting is possible or re-harvesting of donor sites becomes available. Disadvantages of its use include the limited abundance and availability of donors, possible transmission of disease, the eventual rejection by the host and its
\end{abstract}

\section{Introduction}

The gold standard for burn wound coverage remains the autologous split-thickness skin graft. However, severe burn patients lack adequate skin donor sites to resurface their burn woundscalling for the use of skin substitutes to meet the shortfall. Skin substitutes are also referred to as artificial skin. In the broadest sense, a skin substitute is anything that substitutes for any of the skin functions. It may be synthetic or biological (1). However, to be more than just a dressing, a biologic skin substitute should in some way be incorporated into the healing wound as happens with allografts and xenografts (2). Cadaveric grafts and porcine grafts are skin substitutes that have been used clinically for several decades. Cadaveric or live donor skins are termed allografts, or homografts, because they are transplanted from one individual to another within the same species. Pig skin grafts are termed xenografts, or handling, storing, transporting and associated costs of provision. Methods: Between August 2010 and August 2014, five patients underwent live skin allografting without medical immunosuppresion. All patients had deep severe burns of more than $40 \%$ burn surface area. 3 skin donors were patients' mothers while 2 were brothers. Results: Three patients had complete healing not requiring skin autografting. One patient had hyper acute rejection and another had normal rejection and underwent secondary auto grafting. Conclusion: Live skin allografting is a useful skin substitute for severely burnt patients in resource limited areas

Keywords: Burns, Allograft, Immunosuppresion, Family Donor

Ann Afr Surg. 2016;13(2): 77-80.

DOI: http://dx.doi.org/10.4314/aas.v13i2.10

heterografts, because they are transplanted from an organism of one species to that of a different species. The ideal skin substitute is nontoxic, has little or no antigenicity, is immunologically compatible, and does not transmit disease $(2,3)$. Many products and techniques are available to the surgeon to aid in wound coverage and healing. These skin substitutes reduce bacterial count, minimize the loss of water, protein, and electrolytes, restore function; facilitate early motion, and provide coverage of vessels, tendons, and nerves to prevent desiccation. Donor skin may be prepared for use in several ways. They may be treated with glycerol and rapidly frozen with liquid nitrogen or they may be lyophilized and freezedried $(4,5)$. Skin from a live donor does not require complex preparation or preservation. It can be used immediately after harvesting and provides a ready source of skin substitute. The closer the donor is 
related to the patient the lesser the immunological rejection process (6). Both allografts and xenografts are biologic dressings only, are ultimately rejected by the patient's immune system, and need to be removed prior to definitive wound treatment or skin grafting. While xenografts are rejected before undergoing revascularization, allografts initially undergo revascularization but are typically rejected after approximately 10 days because of the strong antigenicity of skin (7).

The host-immune mechanism can be manipulated to prolong the interval from allograft application to rejection. Immunosuppressive agents have been employed to achieve this, including; alloantiserum, azothiaprine, antithymocyte globulin, and cyclosporine (8-13). Cyclosporine extends graft viability by suppressing $\mathrm{T}$ lymphocytes (13). Firstset rejection is prevented by inhibiting donor-specific $\mathrm{T}$ cells, but second-set rejection proceeds in normal fashion, and future grafts from another donor are rejected (14). The techniques of intermingling grafts and immunosuppressants extend the period that allografts can be left in contact with open wounds, allowing more time for regeneration of autogenous donor sites and decreasing the amount of allograft required for cover.

Burns patients are immunosupressed (15-17). The more severe the burn the greater the degree of immunosuppresion. Such burn patients will have rejection of the allografts delayed up to several weeks (18). This phenomenon will obviate the need to use immunosuppressive drugs in severely burnt patients. We took advantage of this fact and performed live skin allograft in five patients without use of immunosuppressive drugs

\section{Methods}

Patients with severe burns of more than $40 \%$ surface area admitted at the Nyeri Provincial General Hospital between August 2010 and August 2014 were included in this study. These patients had been in the wards for more than three months being managed by wound dressing with silver sulfadiazine every 3 days. The wounds were well granulated but patients lacked skin donor sites for auto grafting. For the donors and recipients, a HIV negative status and hemoglobin level above $10 \mathrm{~g} / \mathrm{dl}$ were considered appropriate. Donors were admitted the night before surgery. Surgery was performed in the same operating room by the same team. Skin was harvested from the donor using a Humby knife under spinal anesthesia. Once adequate skin was harvested it was meshed using a surgical blade and grafting performed on the burn patients. The donors were discharged on the first post operative day on oral analgesia and reviewed on the $14^{\text {th }}$ post operative day for dressing removal. The burn patients remained in hospital until fully healed. Ethical approval was obtained from the hospital administration for live allografting for all the patients and informed consent obtained from both patients and donors. In case of minors the parents provided consent.

\section{Results}

Five patients underwent live skin allografting between August 2010 and August 2014. There were three adults and two children. Three were male. Causes of burns included open flames in two and hot water, hot tea and petrol in one patient each. Both children and one adult had the mothers as skin donors (Figure 1), while two adults had their brothers as donors (Figure 2).


\section{Recipient Outcome}

Complete healing not requiring secondary grafting was seen in 3 patients ( 2 children, 1 adult) as shown in Figure 3. One patient had normal acute graft rejection, which completed around 14th post operative day. She however had a well granulated bed and a reduced wound size. She underwent auto grafting on the 21st post operative day. One patient had hyper acute rejection of the allograft (Figure 4). There was more than $90 \%$ graft loss on the 5 th post operative day and complete loss by 8th post operative day. Four out of 5 patients fully recovered and were discharged 
home. One patient had a poor outcome and requested transfer to a hospital near his home for nursing care.

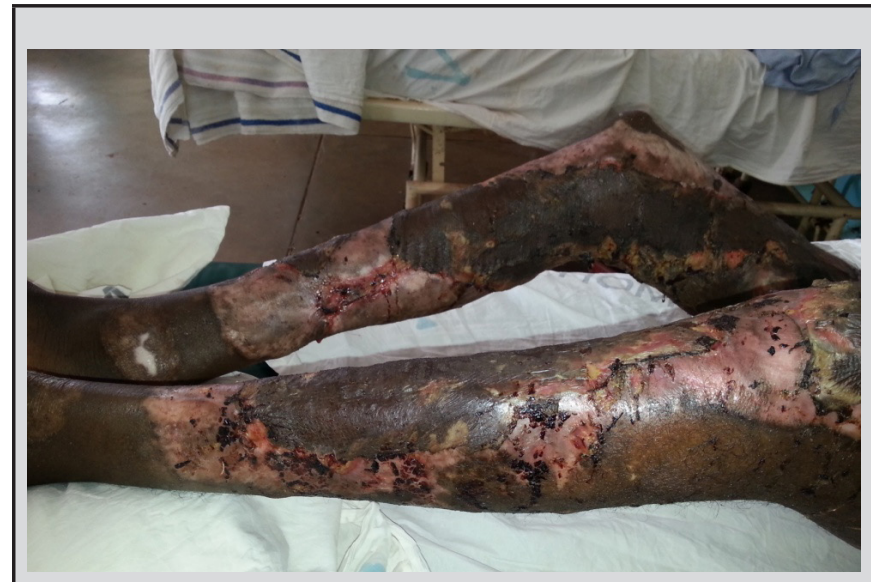

Figure 3: Complete Graft Take at $14^{\text {th }}$ Post Operative Day

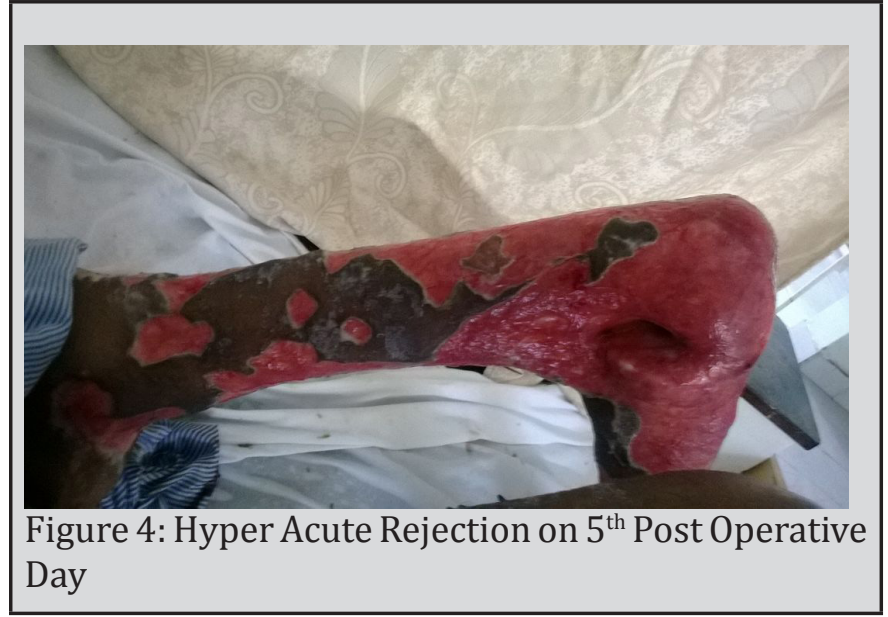

\section{Donor Outcome}

All donor sites showed full granulation on the 14th post operative day. None of the donor sites required dressings after the 14th post operative day. All donor sites were fully healed by 3 months post operative. No incident of keloids or hypertrophic scar was reported

\section{Discussion}

Severe burns of more than $30 \%$ surface area present a great challenge in management especially in rural Kenya. In the developed world, new techniques and devices have contributed to significantly improved functional and aesthetic results. The development of skin substitutes, along with stem cell therapies and tissue engineering that is used for such patients, is out of reach to the majority of rural Kenyan patients. This calls for use of a simple and affordable technique to achieve comparable results.

Cadaveric allografts have been used as temporary skin cover in many parts of the world for many years. Donors are recruited before their death and the skin is harvested immediately after death. The skin is treated with glycerol and rapidly frozen with liquid nitrogen or they may be lyophilized and freeze-dried. It is then banked for later use(19). Skin banking is an expensive undertaking and is not readily available in resource poor countries. There is no known skin bank in East Africa. This coupled with high cost of commercial artificial skin products has denied poor patients access to skin cover for those who need it most.

Live skin allografts can adequately fill this gap. The abundance of donors and minimal pre operative preparation is ideal for the poor countries. It has been used successfully in some countries with good results though with very small number of patients reported (20-22). Acceptability has been retarded by cultural beliefs and fear of contacting diseases (23). However, with adequate pre operative counseling we were able to have full acceptance of the procedure from both donors and recipients. Since allografts are used as temporary skin cover, HLA typing is not necessary. It is expected that the skin will undergo normal acute rejection that starts from 8th day and completes by the 21st day. With immunosuppresion, rejection may be delayed to up to 3 months or more (24). This is adequate time for a partial thickness burn to have healed. In case of a deep burn, the time is sufficient for the bed to have granulated enough for autografting. Patients with severe burns are immunosupressed and have rejection much delayed and hence show good outcome with allografting as demonstrated (25).

\section{Conclusion}

Live skin allograft without medical immunosuppresion is an effective and safe option to be considered for providing skin cover in severe burns in developing countries with limited resources. With good counseling of donors and patients it can have a wide acceptance in our population. It should be part of the surgeon's reconstructive ladder for such extensive burns and can be a useful tool in rural Kenya where little option is available. It is easy to teach and requires minimal resources.

\section{References}

1. Shakespeare P. Burn Wound Healing and Skin Substitutes. Burns. 2001; 27: 517-22

2. Brown AS, Barot LR. Biologic Dressings and Skin Substitutes. Clin Plast Surg. 1986; 13:69

3. Phillips TJ: New Skin for Old. Developments in Biological Skin Substitutes. Arch Dermatol. 1998; $134: 344$

4. Kolenik SA III, Leffell DJ: The Use of Cryopreserved Human Skin Allografts in Wound Healing following Mohs Surgery. Dermatol Surg.1995; 21:61.

5. Hoekstra MJ, Kreis RW, Du Pont JS. History of the Euro Skin Bank: The Innovation of Preservation Technologies. Burns.1994;20:43 
6. Suthanthiran M. Clinical Application of Molecular Biology: A Study of Allograft Rejection with Polymerase Chain Reaction. Am J Med Sci. 1997; 313:264

7. Jignesh U, Justin MS, Stefan S, et al. Relative Antigenicity of Allograft Components and Differential Rejection. Transplantation. 2008; 55:69

8. Dohi K, Asahara T, Ono E, et al. Studies of Alloantibody Effect on Skin Graft Survival in Mice. Hiroshima J Med Sci.1984; 33:271

9. Dohi K, Asahara T, Ono E, et al. Studies of Immunosuppressive Effect of Anti-Recipient Alloantiserum. I. Effect on Skin Graft Survival in Mice. Hiroshima J Med Sci.1984;33:507

10. Steines NA, Gug K, Davies DAL. Passive Enhancement of Mouse Skin Allografts; Specificity of the Antiserum for Major Histocompatibility Complex Antigens. Transplantation.1974;18:192

11. McCullough CS, Sugarbaker PH, Matthews W. Effects of Passive Enhancement on Graft And Host. Transplantation.1984; 37:91

12. Gallico GG III. Biologic Skin Substitutes. Clin Plast Surg.1990; 17:519

13. Towpik E, Kupiec-Weglinski JW, Tyler DS. Cyclosporine and Experimental Skin Allografts: Long-Term Survival in Rats Treated with Low Maintenance Doses. Plast Reconstr Surg.1986; 77:268.

14. Towpik E. Meshed Allograft Skin and Cyclosporin Immunosuppression. Br J Plast Surg.1989; 42:618

15. Robins EV. Immunosuppression of the Burned Patient. Erratum in Crit Care Nurs Clin North Am. 1990; 2:1

16. Barlow Y.T Lymphocytesand Immunosuppression in the Burned Patient: A Review. Burns.1994; 20: 487-90
17. Schwacha MG, Chaudry IH. The Cellular Basis of Post-Burn Immunosuppression: Macrophages and Mediators. Int J Mol Med. 2000; 10:239-43

18. Alexander M, Chaudry IH, Schwacha MG. Relationships Between Burn Size, Immunosuppression, and Macrophage Hyperactivity in a Murine Model of Thermal Injury. Cell Immunol. 2002; 220(1):63-9.

19. Francisco M, Gomez MC, Madnaveitia JA, et al. The Clinical Use of Cryopreseved Human Skin Allograft for Transplantation. Cir Cir. 2015; 6:485-91

20. Converse J, Duchet G. Successful Homologous Skin Grafting in a War Burn Using Identical Twin as Donor. Plastic and Reconstructive surgery. 1947; 4:342-44****

21. Coenen J, Klassen HJ, Sauer. Successful Homografting in an Elderly Patient with Extensive Burns Using his Identical Twin Brother as Donor. Burns. 1990; 16(3):225-26

22. Leodoro B, Chang A, Jitoko K. Live Sibling Allografts for Severe Burns in a Pediatric Patient: A Viable Option in Developing Countries. J Pediatr Surg Case Rep. 2014; 11:498-500

23. Enoch S, Shaaban S, Dunn K.W. Informed Consent Should be Obtained from Patients to Use Products (Skin Substitutes) and Dressings Obtained from Biological Sources. J Med Ethics. 2005; 31: 2-6

24. Benichou G, Yamada Y, Yun SH, et al. Immune Recognition and Rejection of Allogeneic Skin Grafts. Immunotherapy. 2011; 3:757-70

25. Parmanent K, Zetterberg A, Emerudh J, et al. Longterm Immunosuppression in Burned Patients Assessed by In Vitro Neutrophil Oxidative Burst (Phagoburst). Burns. 2007; 7:865-87 